\title{
RESEARCH
}

Open Access

\section{Predicting in-hospital mortality in pneumonia-associated septic shock patients using a classification and regression tree: a nested cohort study}

Jaime L Speiser ${ }^{1}$, Constantine J Karvellas ${ }^{2,3^{*}}$ (D), Geoffery Shumilak ${ }^{4}$, Wendy I Sligl ${ }^{4}$, Yazdan Mirzanejad ${ }^{7}$, Dave Gurka ${ }^{8}$, Aseem Kumar ${ }^{9}$, Anand Kumar ${ }^{5,6}$ and the Cooperative Antimicrobial Therapy of Septic Shock (CATSS) Database Research Group

\begin{abstract}
Background: Pneumonia complicated by septic shock is associated with significant morbidity and mortality. Classification and regression tree methodology is an intuitive method for predicting clinical outcomes using binary splits. We aimed to improve the prediction of in-hospital mortality in patients with pneumonia and septic shock using decision tree analysis.

Methods: Classification and regression tree models were applied to all patients with pneumonia-associated septic shock in the international, multicenter Cooperative Antimicrobial Therapy of Septic Shock database between 1996 and 2015. The association between clinical factors (time to appropriate antimicrobial therapy, severity of illness) and in-hospital mortality was evaluated. Accuracy in predicting clinical outcomes, sensitivity, specificity, and area under receiver operating curve of the final model was evaluated in training $(n=2111)$ and testing datasets $(n=2111)$.

Results: The study cohort contained 4222 patients, and in-hospital mortality was $51 \%$. The mean time from onset of shock to administration of appropriate antimicrobials was significantly higher for patients who died (17.2 h) compared to those who survived (5.0 h). In the training dataset $(n=2111)$, a tree model using Acute Physiology and Chronic Health Evaluation II Score, lactate, age, and time to appropriate antimicrobial therapy yielded accuracy of $73 \%$ and area under the receiver operating curve 0.75 . The testing dataset $(n=2111)$ had accuracy of $69 \%$ and area under the receiver operating curve 0.72 .
\end{abstract}

Conclusions: Overall mortality (51\%) in patients with pneumonia complicated by septic shock is high. Increased time to administration of antimicrobial therapy, Acute Physiology and Chronic Health Evaluation II Score, serum lactate, and age were associated with increased in-hospital mortality. Classification and regression tree methodology offers a simple prognostic model with good performance in predicting in-hospital mortality.

Keywords: Pneumonia, Septic shock, Classification and regression tree, Antimicrobial therapy,

\footnotetext{
* Correspondence: dean.karvellas@ualberta.ca

${ }^{2}$ Department of Critical Care Medicine, University of Alberta, 1-40

Zeidler-Ledcor Building, Edmonton, Alberta T6G-2X8, Canada

${ }^{3}$ Division of Gastroenterology and Hepatology, University of Alberta,

Edmonton, Canada

Full list of author information is available at the end of the article
}

(c) The Author(s). 2018 Open Access This article is distributed under the terms of the Creative Commons Attribution 4.0 International License (http://creativecommons.org/licenses/by/4.0/), which permits unrestricted use, distribution, and reproduction in any medium, provided you give appropriate credit to the original author(s) and the source, provide a link to the Creative Commons license, and indicate if changes were made. The Creative Commons Public Domain Dedication waiver (http://creativecommons.org/publicdomain/zero/1.0/) applies to the data made available in this article, unless otherwise stated. 


\section{Background}

Pneumonia complicated by septic shock is associated with significant morbidity and mortality. It is a leading cause of hospitalization and death with an estimated 423,000 emergency department visits per year and 15.9 deaths per 100,000 individuals in the USA [1,2]. Annual medical costs associated with pneumonia were in excess of $\$ 10$ billion annually in 2011 [3]. Most existing literature in the prognostication of pneumonia is targeted at risk stratification of patients presenting to hospital to determine the optimal location of care by predicting risk of death. Little data exists on predicting in-hospital mortality in patients presenting with pneumonia complicated by septic shock.

The primary aim of this study was to use classification and regression tree (CART) methodology to predict in-hospital mortality of patients with pneumonia complicated by septic shock. CART methodology allows the development of predictive models using binary splits on variables which can be read like a flow chart [4, 5]. Gaining popularity in diverse medical fields [6-8], CART models offer an intuitive method for predicting outcomes by using processes familiar to clinicians (e.g., "high" versus "low" values of a predictor). We hypothesized that CART models predicting in-hospital mortality would have good overall performance in terms of predictive accuracy, sensitivity, specificity, and area under the receiver operating curve (AUROC). Specifically, the objectives for this study were to:

1. Assess overall demographic and clinical characteristics of patients with pneumoniaassociated septic shock

2. Compare demographic and clinical characteristics of pneumonia-associated septic shock patients based on clinical outcomes

3. Develop a CART model containing variables suggested within current literature to predict inhospital mortality for patients with pneumoniaassociated septic shock

4. Assess performance of the CART model using predictive accuracy, sensitivity, specificity, and AUROC

\section{Methods}

This was a nested cohort study within a retrospective database (the Cooperative Antimicrobial Therapy of Septic Shock (CATSS) Database) of patients with septic shock. Data was collected from 28 medical centers in Canada, the USA, and Saudi Arabia between 1996 and 2015. The details of the study design and data collection were described in a previous paper [9]. Approval was obtained from the Institutional Review Boards of all participating institutions. This study was written according to the STROBE Guideline for reporting retrospective studies (see Additional file 1) [10].

\section{Study design: patients and setting}

Clinical and microbiological data was extracted for all patients with pneumonia enrolled in the CATSS database. All patients in the CATSS database had septic shock, so that all patients included in our study had both pneumonia and septic shock. The diagnosis of pneumonia was made at the discretion of the physician and based on clinical, microbiological, and radiographic information. Only patients with a primary diagnosis of pneumonia were eligible for this study. Patient records and information were anonymized and de-identified prior to use in this analysis. Eligible patients with missing outcome data were excluded from the final analysis.

\section{Exposures and outcomes}

Baseline patient characteristics including demographics and comorbid conditions were obtained at enrollment into the registry. Data collected within the first $24 \mathrm{~h}$ of septic shock diagnosis included serum bicarbonate level, serum lactate, bilirubin, creatinine, platelet count, international normalized ratio (INR), white blood cell (WBC) count, number of organ failures, and Acute Physiology And Chronic Health Evaluation II (APACHE II) score [11]. The primary outcome of interest was in-hospital mortality. Time to administration of appropriate antimicrobials was defined as the time of development of shock (hypotension with a mean arterial pressure < $65 \mathrm{mmHg}$ and need for vasopressor support) to the time of receipt of antimicrobial therapy listed in the CATSS registry based on review of original patient records.

\section{Operational definitions}

Septic shock was defined using the 1992 ACCP/SCCM guidelines [12]. Per that definition, patients were required to have documented or suspected infection, persistent hypotension requiring vasopressors, and at least two of the following four elements: (1) a heart rate of > 90 beats/min, (2) a respiratory rate $>20$ breaths/min or arterial partial pressure of carbon dioxide $\left(\mathrm{PaCO}_{2}\right)$ of $<$ $32 \mathrm{mmHg}$. (3) a core temperature of $<36{ }^{\circ} \mathrm{C}$ or $>38{ }^{\circ} \mathrm{C}$, and (4) a WBC count $<4000 / \mu \mathrm{L}$ or $>12,000 / \mu \mathrm{L}$ or bands $>10 \%$. Hypotension was considered to represent the initial onset of septic shock when it persisted despite adequate fluid resuscitation (2 1 of crystalloid) [13]. Predetermined rules were used to define documented and suspected infections and to assign significance to clinical isolates as previously described [9]. Cases of septic shock caused by infections acquired $>48 \mathrm{~h}$ after hospital admission were classified as nosocomial cases.

Predetermined rules were used to assess the appropriateness and delays of initial empiric antimicrobial 
therapy $[9,13,14]$. For septic shock with positive cultures, initial antimicrobial therapy was considered appropriate if an antimicrobial with in vitro activity appropriate for the isolated pathogen or pathogens was the first new antimicrobial agent given after the onset of recurrent or persistent hypotension or was initiated within $6 \mathrm{~h}$ of the administration of the first new antimicrobial agent. Initial therapy not meeting these criteria was considered inappropriate [9]. For septic shock with negative cultures, initial antimicrobial therapy was considered appropriate when an antimicrobial agent consistent with broadly accepted norms for empiric management of the typical pathogens for the clinical syndrome was the new antimicrobial agent given after the onset of recurrent or persistent hypotension or was initiated within $6 \mathrm{~h}$ of administration of the first new antimicrobial agent [9]. The designation of appropriateness of empiric therapy of culture-negative infections was based on recommendations listed in the "Clinical Approach to Initial Choice of Antimicrobial Therapy" from the Sanford Guide to Antimicrobial Therapy (most recently available edition at the time of the case). Additionally, infectious disease physicians and microbiologists were consulted at the discretion of the clinical team to account for local practice patterns and regional bacterial resistance patterns during the study period. To evaluate the predictive performance of the models, specificity is defined as the proportion of correctly predicted outcomes of death and sensitivity is the proportion of correctly predicted outcomes of survival.

\section{CART analysis}

CART is a type of decision tree algorithm which follows deterministic rules to develop prediction models for continuous or categorical outcomes. This is a popular method in clinical prediction modeling because CART offers models that are simple to use with no calculations or computer applications to obtain predictions [6-8]. Additionally, CART models offer clear interpretation by using high versus low values of clinical variables related to the outcome of interest based on optimal splitting criteria from an automated algorithm. Trees are read from top to bottom like a flow chart in order to obtain a prediction for a specified outcome (e.g., survived or died). Starting at the top of a tree, branches corresponding to observed clinical features are followed until a terminal node has been reached and the fraction of patients contained in each outcome group is displayed. These tables may be used to assess the probability that a patient falls within each outcome category.

CART models were developed using the following algorithm first introduced by Breiman [4].Trees were constructed firstly by selecting the variable that optimally separated outcome groups, and a binary split was made.
Then, from both of these subgroups, subsequent variables were selected with replacement (meaning that variables can be used more than once within a model) that optimally separated outcome groups, and second levels of binary splits were made. Variable splits were made recursively until stopping criteria were reached, in which case a terminal node occurred. At each terminal node was the outcome prediction for the specific subset of the data.

The features of CART described in the previous paragraph are advantageous compared to standard logistic regression for modeling binary outcomes. Potential deficiencies of logistic regression for clinical prediction modeling include cumbersome calculations (e.g., inserting numbers and exponentiation requires a calculator or application), unclear interpretation of results (e.g., log odds ratios are not intuitive, especially in the presence of interactions between predictor variables), and unsatisfied assumptions (e.g., linear relationship between predictors and outcome via the link function may be inappropriate). CART also includes a method for handling missing predictor data using surrogate splits while logistic regression requires missing data to be filled in using a separate imputation method for all observations prior to developing a prediction model. For these reasons, CART is a beneficial framework for developing clinical prediction models compared to logistic regression.

\section{Variables}

The main outcome of interest was in-hospital mortality. Multiple variables were used in developing the prediction model. Clinical variables included age, sex, use of mechanical ventilation, location of infection acquisition (nosocomial or community), underlying immunosuppression, number of systems with end-organ dysfunction, time to appropriate antimicrobial therapy, body mass index, and APACHE II score. Biochemical variables included serum lactate, bilirubin, sodium, creatinine, INR, platelets, WBC count, and albumin. Microbiological variables included culture positivity, concomitant bacteremia/fungemia, isolated fungal and bacterial pathogens, and the presence of antimicrobial resistant organisms. All clinical predictors were collected at baseline unless otherwise noted.

\section{Statistical methods}

Analyses were completed using RStudio software [15]. Patient characteristics were presented as mean (standard deviation (SD)) or $n$ (percent) and compared using $t$ tests and binomial tests using the $\mathrm{R}$ package tableone [16]. $P$ values of $<0.05$ were considered statistically significant. CART models were constructed using a training dataset $(n=2111)$ and were assessed using a testing dataset $(n=2111)$. Training and test data were randomly split from the entire dataset. The $\mathrm{R}$ package rpart was 
used to develop the CART models [17]. Missing predictor data were handled using the method of surrogate splitting, which is a standard built-in feature of CART implementation using the rpart package. CART can sometimes produce models, which overfit data (i.e., they can model too many splits for a specific training dataset), which may not predict well for independent test data. One of the ways to reduce overfitting is by constraining the number of observations, which each terminal node of the tree must contain. We required that the minimum number of observations in terminal nodes of the CART was 100 (i.e., the tuning parameter for minimum bucket size was 100) to provide a sufficient amount of data relative to the total training sample for meaningful predictions within the final variable splits. Prediction models were assessed in terms of overall accuracy, sensitivity, and specificity using binomial estimates and confidence intervals. AUROC and its corresponding confidence intervals were determined using the R packages ROCR [18] and cvAUC [19].

\section{Results}

Overall demographic and clinical characteristics

In total, 4222 patients (61\% male) with pneumonia and septic shock were included in the analysis (Table 1$)$. The mean (SD) age of patients was 62 (17) years. Sixty-three percent $(n=2652)$ had positive cultures from clinical isolates, $21 \%(n=876)$ had concomitant bacteremia, and $35 \%(n=1075)$ had nosocomial infections. Of patients with positive cultures, the most common pathogens were Staphylococcus aureus ( $n=702,27 \%)$, Streptococcus spp. $(n=658,25 \%)$, Pseudomonas spp. $(n=267,10 \%)$, Escherichia coli $(n=225,9 \%)$, Klebsiella spp. $(n=183$, $7 \%)$, and Haemophilus influenzae $(n=118,4.4 \%)$. Mean (SD) APACHE II score was 26 (8), and serum lactate was $4.1(3.9) \mathrm{mmol} / \mathrm{L}$ at onset of septic shock. During ICU admission, $89 \%(n=3760)$ required mechanical ventilation. Of 3048 patients who received appropriate antimicrobial therapy after the development of septic shock, the mean time to administration of antimicrobials was $10.9 \mathrm{~h}(\mathrm{SD}=18.6 \mathrm{~h})$. Fifty-one percent $(n=2141)$ of patients died in hospital.

Of patients with pneumonia and septic shock, 2141 died in hospital and 2081 survived. Patients who died in hospital were significantly older (mean age of 65 versus 59 ) and had lower body mass index (28 versus 27 ) when compared to survivors (Table 2). The presence of concomitant bloodstream infection, empyema, positive microbiology, gram-negative pathogens, and fungal pathogens were associated with increased in-hospital mortality. Nosocomial pneumonia infections, higher APACHE II scores, and higher numbers of organ failures were also associated with worse outcomes (Table 2). Mechanical ventilation was more commonly used in patients who died. Admission biochemistry revealed that patients who died had significantly lower platelets, higher lactate, higher INR, higher bilirubin, and lower albumin compared to patients who survived. There was no significant difference detected between the groups for white blood cell count, sodium, and creatinine. In-hospital mortality was significantly more common in patients who were immunocompromised. The mean time to administration of appropriate antimicrobial therapy was $5 \mathrm{~h}$ in patients who survived and $17 \mathrm{~h}$ in patients who died.

\section{CART model predicting in-hospital mortality}

The overall dataset was randomly split into training data for model development and testing data for model validation. There were no significant differences detected between the training and test datasets. The CART model for predicting mortality in patients with pneumonia and septic shock is depicted in Fig. 1. Variables included within the model were the time to administration of appropriate antimicrobial therapy, APACHE II score, serum lactate, and age. The most important predictor of in-hospital mortality was the time to appropriate antimicrobial therapy.

The following features were associated with higher probability of death:

1. Time from onset of septic shock to administration of appropriate antimicrobial therapy $>6.6 \mathrm{~h}$ (node 1 , probability of death $=0.76$ )

2. Time from onset of septic shock to administration of appropriate antimicrobial therapy $<6.6 \mathrm{~h}$,

APACHE $>28$, and lactate $>6.3 \mathrm{mmol} / \mathrm{L}$ (node 5 , probability of death $=0.817$ )

3. Time from onset of septic shock to administration of appropriate antimicrobial therapy $<6.6 \mathrm{~h}$, APACHE $>28$, lactate $<6.3 \mathrm{mmol} / \mathrm{L}$, and age $>65$ (node 7 , probability of death $=0.670$ )

The following features were associated with higher probability of survival:

1. Time from onset of septic shock to administration of appropriate antimicrobial therapy $<6.6 \mathrm{~h}$ and APACHE $<28$ (node 4, probability of survival = $0.744)$

2. Time from onset of septic shock to administration of appropriate antimicrobial therapy $<6.6 \mathrm{~h}$,

APACHE $>28$, lactate $<6.3 \mathrm{mmol} / \mathrm{L}$, and age $<65$ (node 8 , probability of survival $=0.591$ )

There were 1174 patients who received appropriate antimicrobials before the onset of septic shock. In the training dataset used to develop the CART prediction model, these were treated as missing. The CART 
Table 1 Demographic and clinical characteristics of pneumonia-associated septic shock patients

\begin{tabular}{|c|c|c|}
\hline & \multicolumn{2}{|c|}{$\begin{array}{l}\text { Overall cohort } \\
(n=4222)\end{array}$} \\
\hline & $N$ & $\begin{array}{l}\text { Number (\%) or mean } \\
\text { (SD) }\end{array}$ \\
\hline \multicolumn{3}{|l|}{ Demographics } \\
\hline Age & 4222 & $62(17)$ \\
\hline Sex (male) & 4222 & $2574(61.0)$ \\
\hline Body mass index & 2013 & $27(8)$ \\
\hline \multicolumn{3}{|l|}{ Microbiology characteristics } \\
\hline Concomitant bloodstream infection & 4222 & $876(20.7)$ \\
\hline Empyema & 4222 & $119(2.8)$ \\
\hline Culture positive & 4222 & $2652(62.8)$ \\
\hline Gram positive & 4222 & $1413(33.5)$ \\
\hline Gram negative & 4222 & $1073(25.4)$ \\
\hline Fungal & 4222 & $20(0.8)$ \\
\hline Hospital-acquired infection & 4222 & $1547(36.6)$ \\
\hline Community-acquired infection & 4222 & $2675(63.4)$ \\
\hline \multicolumn{3}{|l|}{ Organ failure/support } \\
\hline APACHE & 3995 & $26(8)$ \\
\hline Organ failure day 1 & 4222 & $3.8(1.5)$ \\
\hline Mechanical ventilation & 4222 & $3760(89.1)$ \\
\hline \multicolumn{3}{|l|}{ Biochemistry (admission) } \\
\hline WBC & 4031 & $16.3(15.7)$ \\
\hline Platelets & 4046 & $206(136)$ \\
\hline Sodium & 2488 & $137.2(7.1)$ \\
\hline Creatinine & 3829 & $189.9(164.6)$ \\
\hline Lactate & 2804 & $4.1(3.9)$ \\
\hline INR & 3695 & $1.7(1.3)$ \\
\hline Bilirubin & 3544 & $29.9(64.6)$ \\
\hline Albumin & 1506 & $22.7(6.5)$ \\
\hline Immunocompromised & 4222 & $561(13.3)$ \\
\hline $\begin{array}{l}\text { Time delay from shock to appropriate } \\
\text { antimicrobials (hours) }\end{array}$ & 3048 & 10.9 (18.6) \\
\hline Primary outcome: in-hospital mortality & 4222 & $2141(50.7)$ \\
\hline
\end{tabular}

framework uses a method called surrogate splitting in order to handle any missing values, in which non-missing variables are used to make a "surrogate" split for any missing values. Thus, the patients who received appropriate antimicrobials before onset of septic shock were included in the CART model development. For use in practice for new observations of patients, one should follow the branch corresponding to time to appropriate antimicrobials $<6.6$ within Fig. 1 (i.e., proceed to node 2).

\section{Predicting in-hospital mortality: an example}

A patient with pneumonia and septic shock presented at the hospital with the following characteristics: antimicrobials were administered $3 \mathrm{~h}$ after septic shock, APACHE II score of 30, lactate of $10.2 \mathrm{mmol} / \mathrm{L}$, and age of 64. At the start, time to antibiotic administration is less than $6.6 \mathrm{~h}$ (true at node 1), so we follow the right branch to node 2. Next, the APACHE II score is $>28$ is true, so we follow the left branch to node 3 . Then, lactate is $>$ $6.3 \mathrm{mmol} / \mathrm{L}$, so we proceed to the left branch to node 5 . Since there are no nodes under node 5 , this is our final prediction for the model. The probability of death for the patient is 0.817 , and the probability of survival is 0.183 . Therefore, the patient is predicted to die in-hospital.

\section{Assessing performance}

Performance measures and the associated confidence intervals for the CART model are presented in Table 3. In the training dataset, the CART prediction model for mortality yielded overall accuracy of $73 \%$, specificity of $75 \%$, and sensitivity of $71 \%$. The model showed good overall performance, with training dataset AUROC of 0.75 . In the testing dataset, the CART prediction model for mortality yielded accuracy of $69 \%$, specificity of $72 \%$, and sensitivity of $65 \%$. The model had good overall performance, with testing dataset AUROC of 0.72 .

\section{Discussion}

\section{Summary of key results}

In this study, we evaluated a large multi-center cohort of patients with pneumonia complicated by septic shock. Overall mortality (51\%) was high in this population. There were 3048 patients who received appropriate antimicrobial therapy after the development of septic shock with a mean time to appropriate antimicrobial therapy of $10.9 \mathrm{~h}$. Patients who died in the hospital were significantly older and had significantly higher APACHE II scores, number of organ failures, and admission serum lactate. Time to administration of appropriate antimicrobial therapy remained the most important predictor of in-hospital mortality in this population. In the training set $(n=2111)$, a CART model using APACHE II score, lactate, age, and time to appropriate antimicrobial therapy yielded predictive accuracy of $73 \%$, specificity $75 \%$, sensitivity $71 \%$, and AUROC 0.75 . In the testing set $(n=$ 2111 ), the CART model offered predictive accuracy of $69 \%$, specificity $72 \%$, sensitivity $65 \%$, and AUROC 0.72 .

The novelty of the study is the use of classification and regression tree (CART) methodology for the development of a simple, accurate prediction model for outcomes in pneumonia patients with septic shock. CART allows for development of prediction models using binary splits and offers an intuitive method for obtaining predictions of outcome using processes familiar to clinicians (e.g., "high" versus "low" values of a predictor). The nonparametric nature of CART offers results that are simple to use and does not require calculation of use 
Table 2 Demographic and clinical characteristics of pneumonia-associated septic shock patients by mortality

\begin{tabular}{|c|c|c|c|c|c|}
\hline & \multicolumn{2}{|c|}{$\begin{array}{l}\text { Died } \\
(n=2141)\end{array}$} & \multicolumn{2}{|c|}{$\begin{array}{l}\text { Survived } \\
(n=2081)\end{array}$} & \multirow[t]{2}{*}{$P$ value } \\
\hline & $N$ & Number (\%) or mean (SD) & $N$ & Number (\%) or mean (SD) & \\
\hline \multicolumn{6}{|l|}{ Demographics } \\
\hline Age & 2141 & $64.6(15.8)$ & 2081 & $58.8(16.7)$ & $<0.001$ \\
\hline Sex (male) & 2141 & $1323(61.8)$ & 2081 & $1251(60.1)$ & 0.277 \\
\hline Body mass index & 974 & $26.6(7.8)$ & 1039 & $27.7(7.7)$ & 0.001 \\
\hline \multicolumn{6}{|l|}{ Microbiology characteristics } \\
\hline Concomitant bloodstream infection & 2141 & $484(22.6)$ & 2081 & $392(18.8)$ & 0.003 \\
\hline Empyema & 2141 & $45(2.1)$ & 2081 & $74(3.6)$ & 0.010 \\
\hline Culture positive & 2141 & $1421(66.4)$ & 2081 & $1231(59.2)$ & $<0.001$ \\
\hline Gram positive & 2141 & $696(32.5)$ & 2081 & $717(34.5)$ & 0.191 \\
\hline Gram negative & 2141 & $608(28.4)$ & 2081 & $465(22.3)$ & $<0.001$ \\
\hline Fungal & 2141 & $16(0.7)$ & 2081 & $4(0.2)$ & 0.009 \\
\hline Hospital-acquired infection & 2141 & $957(44.7)$ & 2081 & $590(28.4)$ & $<0.001$ \\
\hline \multicolumn{6}{|l|}{ Organ failure/support } \\
\hline APACHE & 2034 & $28.5(8.0)$ & 1961 & $22.8(6.7)$ & $<0.001$ \\
\hline Organ failure day 1 & 2141 & $4.2(1.6)$ & 2081 & $3.4(1.3)$ & $<0.001$ \\
\hline Mechanical ventilation & 2141 & 2005 (93.6) & 2081 & $1755(84.3)$ & $<0.001$ \\
\hline \multicolumn{6}{|l|}{ Biochemistry (admission) } \\
\hline WBC & 2052 & $16.3(17.9)$ & 1979 & $16.4(13.0)$ & 0.757 \\
\hline Platelets & 2021 & $195(143)$ & 2025 & $216(128)$ & $<0.001$ \\
\hline Sodium & 1121 & $137.4(7.2)$ & 1367 & $137.0(7.0)$ & 0.192 \\
\hline Creatinine & 1937 & $192.2(164.6)$ & 1892 & $187.5(164.6)$ & 0.377 \\
\hline Lactate & 1447 & $5.1(4.6)$ & 1357 & $3.1(2.8)$ & $<0.001$ \\
\hline INR & 1848 & $1.9(1.5)$ & 1847 & $1.6(1.1)$ & $<0.001$ \\
\hline Bilirubin & 1768 & $39.7(82.7)$ & 1776 & $20.1(36.7)$ & $<0.001$ \\
\hline Albumin & 621 & $21.6(6.3)$ & 885 & $23.5(6.4)$ & $<0.001$ \\
\hline Immunocompromised & 2141 & $360(16.8)$ & 2081 & $201(9.7)$ & $<0.001$ \\
\hline Time delay from shock to appropriate antimicrobials (hours) & 1494 & $17.2(23.6)$ & 1554 & $5.0(5.6)$ & $<0.001$ \\
\hline
\end{tabular}

of an application. Models are easily read and interpreted using a flow chart diagram. These aspects of CART are advantageous compared to logistic regression, where calculations may be cumbersome (e.g., plugging in numbers and exponentiation requires a calculator or application), interpretation of results may be unclear (e.g., if there are interactions between two or more predictors), and assumptions may not be satisfied.

\section{Comparison with the literature}

Patients with pneumonia complicated by septic shock are at substantial risk of poor outcomes. The 51\% in-hospital mortality observed in this cohort is substantially higher than the reported mortality for population-level outcomes in patients with pneumonia and patients presenting with pneumonia and septic shock [20, 21]. Despite these studies, a lack of data on predicting outcomes for patients with pneumonia and septic shock remained.

In this study, a mechanism for predicting the probability of in-hospital mortality was developed using CART methodology. Previous prediction models have focused on predicting patient outcomes for purposes of risk stratification at presentation to hospital with pneumonia [22-24]. Consistent with previous literature, our study highlights that the presence of septic shock and the severity of illness (APACHE II), age, lactate, and time to administration of appropriate antimicrobial therapy significantly impacts survival in patients with pneumonia. In our study, multivariable CART analysis demonstrated that the most important predictor of mortality was the increasing time from onset of septic shock to 


\section{Start $(\mathrm{N}=2111)$}

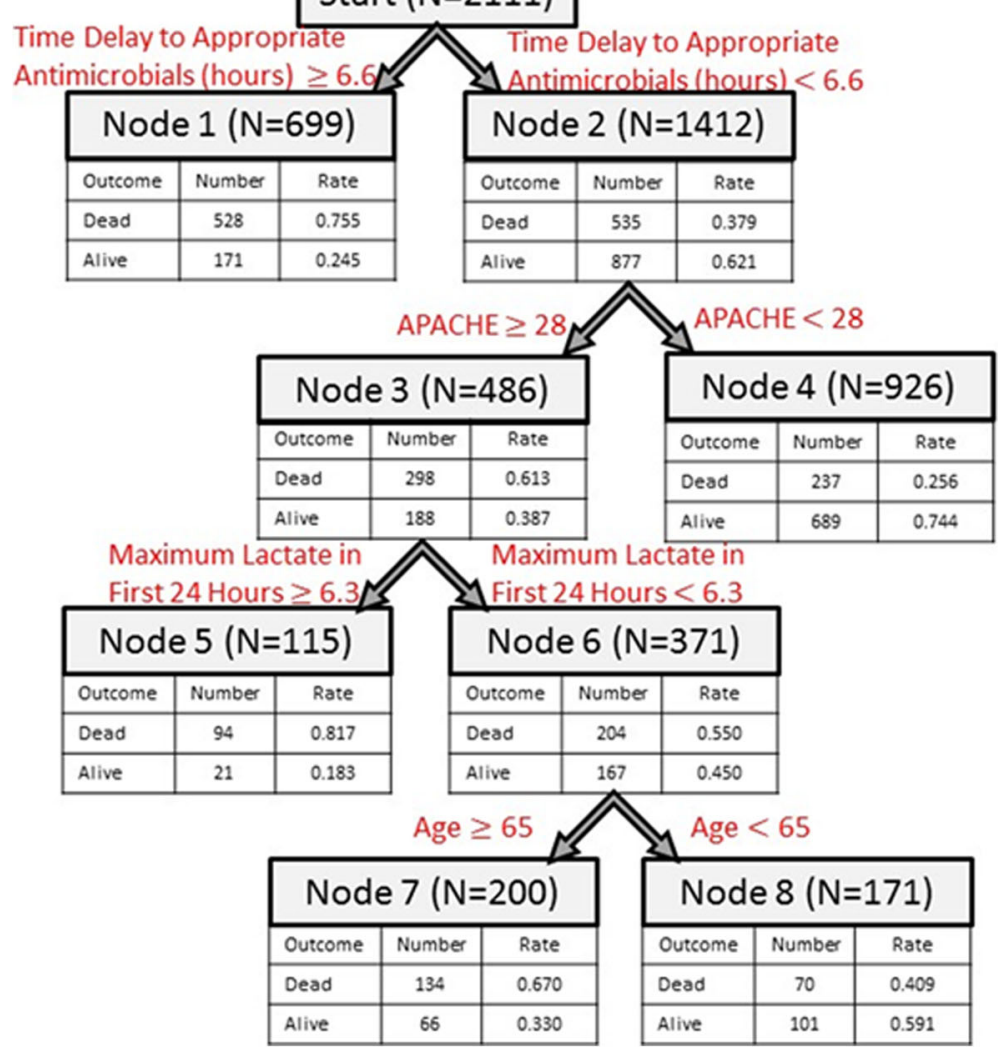

Fig. 1 Depicts the resulting classification and regression tree for predicting in-hospital mortality. The decision tree contains four predictors: time to appropriate antimicrobial therapy, APACHE II score, lactate, and age. Terminal nodes containing predictions for new observations include 1, 5, and 7 (predict death) and 4 and 8 (predict alive). To obtain a prediction, one starts at the top of the tree and follows the arrow corresponding to data for the new observation until a terminal node is reached

administration of appropriate antimicrobial therapy. Additional predictors of in-hospital mortality included severity of illness (APACHE II score), high serum lactate, and older age. Our study complements previous research by highlighting the importance of early intervention and administration of appropriate antimicrobial therapy to optimize outcomes in patients with septic shock.

Though CART models have existed for several decades, there is a paucity of decision tree models available for predicting outcomes in critically ill patient populations. Wong et al. [25] use CART to analyze 355 children with septic shock to assess biomarkers and clinical variables. The resulting decision tree consisted of five

Table 3 Performance measures (95\% exact binomial confidence intervals) for the CART model prediction in-hospital mortality

\begin{tabular}{lllll}
\hline Model & $\begin{array}{l}\text { Accuracy } \\
(95 \% \mathrm{Cl})\end{array}$ & $\begin{array}{l}\text { Specificity } \\
(95 \% \mathrm{Cl})\end{array}$ & $\begin{array}{l}\text { Sensitivity } \\
(95 \% \mathrm{Cl})\end{array}$ & $\begin{array}{l}\text { AUROC } \\
(95 \% \mathrm{Cl})\end{array}$ \\
\hline Training & 0.73 & 0.75 & 0.71 & 0.75 \\
$(n=2111)$ & $(0.71,0.75)$ & $(0.73,0.78)$ & $(0.68,0.74)$ & $(0.73,0.78)$ \\
Testing & 0.69 & 0.72 & 0.65 & 0.72 \\
$(n=2111)$ & $(0.67,0.71)$ & $(0.70,0.75)$ & $(0.62,0.68)$ & $(0.69,0.75)$ \\
\hline
\end{tabular}

biomarker-based decision rules with ten variable splits. This work was primarily done to complement microarray work to explore potential gene products as targets in sepsis. Wong et al. subsequently applied the same five biomarkers along with lactate, age, and chronic disease status [26] in 672 adult patients with septic shock with and developed a clinical prediction model with an area under the receiver curve of 0.72 (validation set), similar to this study. Besides these two studies which primarily focused on gene products/potential novel biomarkers (both $<700$ patients), the decision tree approach for prediction has not been previously used for a large population of adults with septic shock using readily available clinical information as in this study.

\section{Limitations}

This study should be interpreted within the limitations of its design. This study is a retrospective analysis of prospectively collected data and only association, not causation, can be inferred. Given that this study was observational, we are unable to conclusively exclude sources of selection bias [27]. We implemented an 
internal validation scheme that used randomly split training and testing datasets to build and evaluate the CART prediction model for mortality. External data should be used to further validate the CART model. Another limitation is that the average time to appropriate antimicrobial therapy was $6 \mathrm{~h}$, which is greater than the current suggested 3-h completion of treatment. These guidelines changed over the course of the study period (from 1996 to 2016), so we included all data in order to have a larger sample size to develop a prediction model. Though current guidelines suggest completion of the sepsis bundle within $3 \mathrm{~h}$ [28], approximately one third of the patients in this study received appropriate antibiotic after $6 \mathrm{~h}$. There are several reasons for the longer time to antibiotics: our study included appropriate use of antibiotics not just time to any usage of antibiotics, about half of the patients included in our study were ward patients which have higher time to appropriate antimicrobials compared to emergency room admissions, and the data range for our study is from 1996 to 2016 during which the time to antibiotics was substantially longer than the standard practice now. Despite these limitations, the strengths include inclusion of patients from 28 intensive care units across three geographic regions.

Limitations of CART modeling include the challenge of determining parameters for model building (e.g., deciding the minimum bucket size) and the possible variability of CART models, as discussed in statistical literature (e.g., [8, 29-32]) (Additional file 2). Inclusion of variables for age and APACHE II, which also used age for its calculation, highlights the importance of age for predicting outcomes of pneumonia-associated septic shock patients. Unlike traditional regression models, which can be negatively influenced by correlated variables, the CART model can adequately handle correlated variables due to the binary nature of splitting. However, these limitations are minimal compared to the beneficial simplicity and relatively high predictive accuracy of CART models.

\section{Conclusion}

Overall mortality in patients with pneumonia and septic shock is high (51\% in the CATSS dataset). Increasing time to appropriate antimicrobial therapy, APACHE II score, serum lactate, and age were significantly associated with in-hospital mortality. CART models offer simple prognostic models with good performance.

\section{Additional files}

Additional file 1: STROBE guideline for reporting retrospective studies. (DOCX 38 kb)

Additional file 2: Benefits of CART, tree development, and limitations of CART models. (DOCX $17 \mathrm{~kb}$ )

\section{Abbreviations}

APACHEIl: Acute Physiology and Chronic Health Evaluation II score; AUROC: Area under the receiver operating curve; CART: Classification and regression tree; CATSS: Cooperative Antimicrobial Therapy of Septic Shock; $\mathrm{Cl}$ : Confidence interval; ICU: Intensive care unit; INR: International normalized ratio; SD: Standard deviation; WBC: White blood cell

The Cooperative Antimicrobial Therapy of Septic Shock (CATSS) Database Research Group

Yaseen Arabi, MD, King Saud Bin Abdulaziz University for Health Sciences, Riyadh, Saudi Arabia

Phillip Dellinger, MD, Cooper Hospital/University Medical Center, Camden NJ, USA

Sandra Dial, MD, McGill University, Montreal QC, Canada

Peter Dodek, MD, University of British Columbia, Vancouver BC, Canada

Paul Ellis, MD, University Health Network, Toronto, ON, Canada

Daniel Feinstein, MD, Moses H. Cone Memorial Hospital, Greensboro NC, USA

Dave Gurka, MD, Rush-Presbyterian-St. Luke's Medical Center, Chicago IL, USA Jose Guzman, MD, Cleveland Clinic, Cleveland, OH, USA

Sean Keenan' MD, Royal Columbian Hospital, New Westminster BC, Canada

Andreas Kramer, MD, Foothills Hospital, Calgary AB, Canada

Aseem Kumar, PhD, Laurentian University, Sudbury, ON, Canada

Stephen Lapinsky, MD, Mount Sinai Hospital, Toronto ON, Canada

Denny Laporta, MD, Jewish General Hospital, Montreal QC, Canada

Bruce Light, MD, Winnipeg Regional Health Authority, Winnipeg MB, Canada

Dennis Maki, MD, University of Wisconsin Hospital and Clinics, Madison WI,

USA

Greg Martinka, MD, Richmond General Hospital, Richmond BC, Canada

Yazdan Mirzanejad, MD, Surrey Memorial Hospital, Surrey, BC, Canada

Joseph E. Parrillo, MD, Hackensack University Medical Centre, Hackensack, NJ, USA

Gourang Patel, PharmD, Rush-Presbyterian-St. Luke's Medical Center, Chicago IL, USA

Brian Bookatz, MD, Brandon General Hospital, Brandon MD, Canada Dan Roberts, MD, Winnipeg Regional Health Authority, Winnipeg MB, Canada John Ronald, MD, Nanaimo Regional Hospital, Nanaimo BC, Canada Dave Simon, MD, Rush-Presbyterian-St. Luke's Medical Center, Chicago IL, USA

Yoanna Skrobik, MD, Hôpital Maisonneuve Rosemont, Montreal QC, Canada Gordon Wood, MD, Royal Jubilee Hospital/Victoria General Hospital, Victoria BC, Canada

Kenneth E. Wood, DO, University of Maryland Medical System, Baltimore MD USA

Associate Members of the CATSS Database Research Group

Muhammed Wali Ahsan, MD, Winnipeg MB, Canada

Mozdeh Bahrainian, MD, Madison W

Rob Bohmeier, MD, University of Manitoba, Winnipeg MB, Canada Lindsey Carter, MD, University of Manitoba, Winnipeg MB, Canada Harris Chou, BSC, of British Columbia, Vancouver BC, Canada

Sofia Delgra, RN, King Saud Bin Abdulaziz University for Health Sciences,

Riyadh, Saudi Arabia

Collins Egbujuo, MD, Winnipeg MB, Canada

Winnie Fu, MD, University of British Columbia, Vancouver BC, Canada

Catherine Gonzales, RN, King Saud Bin Abdulaziz University for Health

Sciences, Riyadh, Saudi Arabia

Harleena Gulati, MD, University of Manitoba, Winnipeg MB, Canada Oliver Gutierrez, MD, University of Manitoba, Winnipeg MB, Canada Aparna Jindal, MD, University of Manitoba, Winnipeg MB, Canada Erica Halmarson, MD, University of Manitoba, Winnipeg MB, Canada Ziaul Haque, MD, Montreal QC, Canada

Johanne Harvey, RN, Hôpital Maisonneuve Rosemont, Montreal QC, Canada

Ehsan Koohpayehzadeh Esfahani, MD, University of Manitoba, Winnipeg MB, Canada

Farah Khan, MD, Toronto ON, Canada

Laura Kolesar, RN, St. Boniface Hospital, Winnipeg MB, Canada

Laura Kravetsky, MD, University of Manitoba, Winnipeg MB, Canada

Runjun Kumar, BSc, Washington University Medical School, St. Louis, MO,

USA

Nasreen Merali, MD, Winnipeg MB, Canada

Sheri Muggaberg, MD, University of Manitoba, Winnipeg MB, Canada

Heidi Paulin, MD, University of Toronto, Toronto ON, Canada 
Cheryl Peters, RN, MD, University of Manitoba, Winnipeg MB, Canada Jody Richards, RN, Camosun College, Victoria BC, Canada Honorata Serrano, RN, King Saud Bin Abdulaziz University for Health Sciences, Riyadh, Saudi Arabia

Amrinder Singh, MD, Winnipeg MB Canada

Katherine Sullivan, MD, University of Manitoba, Winnipeg MB, Canada Robert Suppes, MD, University of Manitoba, Winnipeg MB, Canada Leo Taiberg, MD, Rush Medical College, Chicago IL, USA

Ronny Tchokonte, MD, Wayne State University Medical School, Detroit MI, USA Omid Ahmadi Torshizi, MD, Montreal QC, Canada

Kym Wiebe, RN, St. Boniface Hospital, Winnipeg MB, Canada

\section{Availability of data and materials}

The datasets analyzed in the current study are available from the senior author (Anand Kumar) on reasonable request.

\section{Authors' contributions}

JLS performed the statistical and data analysis and drafted and extensively revised the final manuscript. CJK conceived the idea of the study and drafted and extensively revised the final manuscript. GS and WIS drafted and extensively revised the final manuscript. YM, DG, and AK collected the data and revised the final manuscript. AK is the principal investigator and responsible for CATSS database who developed the initial registry; provided content expertise, significant guidance on compilation of the database, and analysis and interpretation of data; and assisted extensively with the manuscript revision. All authors read and approved the final manuscript.

\section{Ethics approval and consent to participate}

Approval was obtained from the Institutional Review Boards of all participating institutions. A waived consent protocol was approved by the health ethics boards of the University of Manitoba and at each individual participating center.

\section{Consent for publication}

Not applicable.

\section{Competing interests}

The authors declare that they have no competing interests.

\section{Publisher's Note}

Springer Nature remains neutral with regard to jurisdictional claims in published maps and institutional affiliations.

\section{Author details}

'Department of Biostatistical Sciences, Division of Public Health Sciences, Wake Forest School of Medicine, Winston-Salem, NC, USA. ${ }^{2}$ Department of Critical Care Medicine, University of Alberta, 1-40 Zeidler-Ledcor Building, Edmonton, Alberta T6G-2X8, Canada. ${ }^{3}$ Division of Gastroenterology and Hepatology, University of Alberta, Edmonton, Canada. ${ }^{4}$ Division of Critical Care Medicine and Infectious Diseases, University of Alberta, Edmonton, Canada. ${ }^{5}$ Section of Critical Care Medicine, University of Manitoba, Winnipeg, Canada. 'Section of Infectious Diseases, University of Manitoba, Winnipeg, Canada. ${ }^{7}$ Surrey Hospital, Surrey, BC, Canada. ${ }^{8}$ Rush Medical College, Chicago, IL, USA. ${ }^{9}$ Laurentian University, Sudbury, ON, Canada.

\section{Received: 26 June 2018 Accepted: 27 September 2018}

\section{Published online: 12 October 2018}

\section{References}

1. Kochanek KD, Murphy SL, Xu J, Tejada-Vera B. Deaths: final data for 2014 National vital statistics reports: from the Centers for Disease Control and Prevention, National Center for Health Statistics. Natl Vital Stat Syst. 2016; 65(4):1-122.

2. Rui P, Kang K. National Hospital Ambulatory Medical Care Survey: 2014 emergency department summary tables. Centers for Disease Control and Prevention; 2014.

3. Pfuntner A, Wier LM, Steiner C. Costs for hospital stays in the United States, 2011: statistical brief\# 168. 2006.

4. Breiman L, Friedman JH, Olshen RA, Stone CJ. Classification and regression trees. Monterrey, CA: Wadsworth and Brooks; 1984.
5. Loh WY. Fifty years of classification and regression trees. Int Stat Rev. 2014; 82(3):329-48.

6. Aguiar FS, Almeida LL, Ruffino-Netto A, Kritski AL, Mello FC, Werneck GL. Classification and regression tree (CART) model to predict pulmonary tuberculosis in hospitalized patients. BMC Pulm Med. 2012;12:40.

7. Garzotto M, Beer TM, Hudson RG, et al. Improved detection of prostate cancer using classification and regression tree analysis. J Clin Oncol. 2005; 23(19):4322-9.

8. Speiser JL, Lee WM, Karvellas CJ. Predicting outcome on admission and post-admission for acetaminophen-induced acute liver failure using classification and regression tree models. PLoS One. 2015;10(4):e0122929.

9. Kumar A, Ellis $P$, Arabi $Y$, et al. Initiation of inappropriate antimicrobial therapy results in a fivefold reduction of survival in human septic shock. Chest. 2009;136(5):1237-48.

10. von Elm E, Altman DG, Egger M, et al. Strengthening the Reporting of Observational Studies in Epidemiology (STROBE) statement: guidelines for reporting observational studies. BMJ. 2007;335(7624):806-8.

11. Knaus WA, Draper EA, Wagner DP, Zimmerman JE. APACHE II: a severity of disease classification system. Crit Care Med Oct. 1985;13(10):818-29.

12. Bone RC, Balk RA, Cerra FB, et al. Definitions for sepsis and organ failure and guidelines for the use of innovative therapies in sepsis. The ACCP/SCCM Consensus Conference Committee. American College of Chest Physicians/ Society of Critical Care Medicine. Chest. 1992;101(6):1644-55.

13. Kumar A, Roberts $D$, Wood KE, et al. Duration of hypotension before initiation of effective antimicrobial therapy is the critical determinant of survival in human septic shock. Crit Care Med Jun. 2006;34(6):1589-96.

14. Kumar A, Zarychanski R, Light B, et al. Early combination antibiotic therapy yields improved survival compared with monotherapy in septic shock: a propensity-matched analysis. Crit Care Med Sep. 2010;38(9): 1773-85.

15. RStudio Team. RStudio. Integrated development for R. RStudio, Inc. Boston, MA URL https://www.rstudio.com; 2015.

16. Yoshida K, Bohn J. tableone: Create "table 1" to describe baseline characteristics. R package version 2015 0. 7. 3;

17. Therneau TM, Atkinson EJ. An introduction to recursive partitioning using the Rpart routines. Rochester: Mayo Foundation; 1997. MN

18. Sing T, Sander O, Beernwinkel N, Lengauer T. ROCR: visualizing classifier performance in R. Bioinformatics. 2005:21(20):3940-1.

19. LeDell E, Petersen M, van der Laan M, LeDell ME. Package 'CvAUC'. 2012.

20. Control CfD, Prevention. Compressed mortality file 1999-2013. CDC wonder on-line database, compiled from compressed mortality file 1999-2013 series 20 no. 2s, 2015. 2015.

21. Garcia-Vidal C, Ardanuy C, Tubau F, et al. Pneumococcal pneumonia presenting with septic shock: host-and pathogen-related factors and outcomes. Thorax. 2009;2009:123612 thx.

22. Fine MJ, Auble TE, Yealy DM, et al. A prediction rule to identify low-risk patients with community-acquired pneumonia. N Engl J Med. 1997;336(4): 243-50

23. Lim W, Van der Eerden M, Laing R, et al. Defining community acquired pneumonia severity on presentation to hospital: an international derivation and validation study. Thorax. 2003:58(5):377-82.

24. Myint PK, Kamath AV, Vowler SL, Maisey DN, Harrison BD. Severity assessment criteria recommended by the British Thoracic Society (BTS) for community-acquired pneumonia (CAP) and older patients. Should SOAR (systolic blood pressure, oxygenation, age and respiratory rate) criteria be used in older people? A compilation study of two prospective cohorts. Age Ageing. 2006;35(3):286-91.

25. Wong HR, Salisbury S, Xiao Q, et al. The pediatric sepsis biomarker risk model. Crit Care. 2012;16(5):R174.

26. Wong HR, Lindsell CJ, Pettilä V, et al. A multibiomarker-based outcome risk stratification model for adult septic shock. Crit Care Med. 2014;42(4):781.

27. Connors AF Jr. Pitfalls in estimating the effect of interventions in the critically ill using observational study designs. Crit Care Med Jun. 2001;29(6): 1283-4.

28. Rhodes A, Evans LE, Alhazzani W, et al. Surviving sepsis campaign: international guidelines for management of sepsis and septic shock: 2016 Intensive Care Med. 2017:43(3):304-77.

29. Chun FKH, Karakiewicz PI, Briganti A, et al. A critical appraisal of logistic regression-based nomograms, artificial neural networks, classification and regression-tree models, look-up tables and risk-group stratification models for prostate cancer. BJU Int. 2007;99(4):794-800. 
30. Hastie T, Tibshirani R, Friedman J, Hastie T, Friedman J, Tibshirani R. The elements of statistical learning. Vol 2. Paolo Alto: Springer; 2009.

31. Lemon SC, Roy J, Clark MA, Friedmann PD, Rakowski W. Classification and regression tree analysis in public health: methodological review and comparison with logistic regression. Ann Behav Med. 2003;26(3):172-81.

32. Province MA, Shannon W, Rao D. 19 classification methods for confronting heterogeneity. Adv Genet. 2001;42:273-86.

Ready to submit your research? Choose BMC and benefit from:

- fast, convenient online submission

- thorough peer review by experienced researchers in your field

- rapid publication on acceptance

- support for research data, including large and complex data types

- gold Open Access which fosters wider collaboration and increased citations

- maximum visibility for your research: over $100 \mathrm{M}$ website views per year

At $\mathrm{BMC}$, research is always in progress.

Learn more biomedcentral.com/submissions 